\title{
Effects of Capsaicin on Intestinal Cephalexin Absorption in Rats
}

\author{
Yukiko Komori, Tetsuya Aiba, Risa SugiYama, Chie Nakai, Hiromu Kawasaki, and \\ Yuji KuROSAKI* \\ Graduate School of Medicine, Dentistry and Pharmaceutical Sciences, Okayama University; Okayama 700-8530, Japan. \\ Received September 24, 2006; accepted December 15, 2006; published online December 18, 2006
}

The effects of capsaicin on intestinal cephalexin absorption were investigated by means of in situ single pass perfusion in rats to clarify whether this pungent compound present in spice is a potential factor altering the intestinal drug absorption processes. Under the control condition, cephalexin was absorbed at a rate of $1.16 \pm 0.08$ and $0.90 \pm 0.06 \mathrm{nmol} / \mathrm{min} / \mathrm{cm}$ in the jejunum and ileum, respectively. The intestinal cephalexin absorption rate was decreased when capsaicin was dissolved in the perfusate at a concentration of $400 \mu \mathrm{M}$, being $0.54 \pm 0.07$ and $0.46 \pm 0.10 \mathrm{nmol} / \mathrm{min} / \mathrm{cm}$ in the jejunum and ileum, respectively. The inhibitive effect of capsaicin on intestinal cephalexin absorption was diminished when ruthenium red, a non-selective inhibitor of the transient receptor potential (TRP) cation channels, was intravenously infused into the rat during the experiment. Moreover, when we evaluated the paracellular permeability of cephalexin by utilizing a competitive inhibitor, glycylsarcosine, it was demonstrated that glycylsarcosine-insensitive intestinal cephalexin absorption in the jejunum was increased by 4.5 times in the presence of $400 \mu \mathrm{M}$ capsaicin. These findings indicate that capsaicin affects both transcellular and paracellular pathways of intestinal cephalexin absorption by interacting with the TRP cation channels in intestinal tissues, in which capsaicin seems to change the transport activity of $\mathrm{H}^{+} /$peptide $^{2}$ co-transporter 1 (PEPT1), and to a lesser degree, it seems to alter the paracellular permeability of the intestinal epithelia.

Key words capsaicin; cephalexin; absorption; transient receptor potential (TRP); peptide co-transporter (PEPT)1; paracellular permeability

Capsaicin (8-methyl- $N$-vanillyl-6-ninenamide) is the compound responsible for the pungent character of various hot peppers (Capsicum), and it is famous for its pungent taste, burning sensation, and various effects on body temperature and gastric acid secretion. ${ }^{1,2)}$ These features of capsaicin are considered to be generated by capsaicin associating with the capsaicin receptor, or the transient receptor potential cation channel of vanilloid type 1 (TRPV1) on the nerve endings of afferent neurons. ${ }^{3-5}$ ) Since it is reported that TRPV1 regulates cation influx in nerve cells and that $\mathrm{Ca}^{2+}$ permeability in those cells largely depends on TRPV1 activity, ${ }^{3,6}$ capsaicin is thought to induce an extracellular $\mathrm{Ca}^{2+}$ influx into nerve cells by associating with TRPV1, and thereby it stimulates afferent neurons to generate the characteristic sensations.

It is reported that the capsaicin-induced $\mathrm{Ca}^{2+}$ influx via TRPV1 prompts the afferent neurons innervating the gastrointestinal tract to release neuropeptides such as calcitonin gene-related peptide (CGRP) and vasoactive intestinal polypeptide (VIP). ${ }^{7)}$ Since these neuropeptides are involved in the regulation and/or maintenance of various gastrointestinal functions by stimulating mesenteric blood flow, preventing mucosal injury, and controlling electrolyte secretion, ${ }^{8-11)}$ capsaicin in savory cuisine is likely to vary the gastrointestinal conditions, and as a result, it may alter the intestinal absorption processes of orally administered medicines. In fact, capsaicin is suggested to affect mucosal integrity, ${ }^{7,12,13)}$ peristaltic activity ${ }^{14)}$ and the absorption/secretion of various electrolytes in the gastrointestinal tract. ${ }^{15)}$ In addition, it was demonstrated that capsaicin decreased intestinal alanine absorption by stimulating afferent neurons to release CGRP and VIP. ${ }^{16-18)}$

Recently, it was suggested that TRPV1 is expressed not only in nerve cells, but also in gastric mucosal tissues and in- testinal epithelia. ${ }^{19-22)}$ As capsaicin associates with TRPV1, thus increasing $\mathrm{Ca}^{2+}$ influx, it may alter the intracellular $\mathrm{Ca}^{2+}$ concentration in these cells. If this is the case, capsaicin probably affects the intestinal drug absorption processes in a direct manner. This is because changes in the intracellular $\mathrm{Ca}^{2+}$ concentration alter the paracellular permeability of intestinal epithelia by modifying the integrity of the epithelial tight junction. ${ }^{23)}$ In addition to the paracellular pathway of the intestinal drug absorption processes, capsaicin may affect the transcellular pathway of the process, since the intracellular $\mathrm{Ca}^{2+}$ concentration is related to the drug transporting activity of various transporters including $\mathrm{H}^{+}$/peptide co-transporter 1 (PEPT1). ${ }^{24)}$ It is therefore suggested with these findings and considerations that capsaicin has an impact on the intestinal drug absorption processes. However, little is known about the effect of capsaicin on intestinal drug absorption.

To clarify whether capsaicin affects intestinal drug absorption, we examined the intestinal drug absorption profiles of rats in the presence and absence of capsaicin in this study, in which we used cephalexin as a model drug since its absorption processes have been thoroughly studied. ${ }^{25,26)} \mathrm{We}$ also examined whether TRPV1 plays an important role in capsaicin's effect, and further elucidated an underlying mechanism of the capsaicin-induced alteration of intestinal cephalexin absorption.

\section{MATERIALS AND METHODS}

Materials Capsaicin and ruthenium red were obtained from Nacalai Tesque (Kyoto, Japan). Cephalexin hydrate or cefalexin hydrate (INN), glycylsarcosine, and FITC-dextran (M.W. 40,000) were purchased from Sigma-Aldrich (St. Louis, MO, U.S.A.). All other chemicals were of the finest grade available. 
Animals Male Wistar rats (190-240 g) were purchased from Japan SLC Inc. (Hamamatsu, Japan). All animal experiments were performed in accordance with the guidelines for animal experimentation of Okayama University.

In Situ Single Pass Perfusion Experiments Prior to the experiments, rats were fasted overnight with free access to water. On the experiment day, rats were anesthetized by ethyl carbamate $(1 \mathrm{~g} / \mathrm{kg}$, i.p. $)$ and fixed on their back. A midline incision was then carefully made on the abdomen and the whole small intestine including the cecum was exposed. After that, the surface of the exposed intestine was softly rinsed with Ringer's solution, and the duodenum and bile duct were ligated. Then, a $10-\mathrm{cm}$ segment of the upper part of the jejunum was chosen to be cannulated. That is, both ends of the jejunum segment were carefully ligated without damaging blood vessels surrounding the segment, and then a silicone tube ( $3 \mathrm{~mm}$ I.D. $-5 \mathrm{~mm}$ O.D.) was inserted into the jejunum segment at its upper and lower ends, respectively. After food debris in the lumen was gently washed out by Ringer's solution, the silicone tube at the upper end of the segment was connected to a syringe infusion pump (Model 22, Harvard apparatus, Holliston, MA, U.S.A.). The other silicone tube at the lower part of the segment was connected to a fraction collector. Following these preparations for the jejunum segment, the ileum segment was also cannulated in the same manner as described above. In brief, a 10-cm segment of the lower part of the ileum, which is adjacent to the cecum, was chosen to be cannulated. Both ends of the ileum segment were ligated and then cannulated with silicone tubes. The lumen was washed and the tubes were connected to another syringe pump (Model 22) and fraction collector, respectively. With these preparations, we could simultaneously perfuse both the jejunum and ileum segments in an independent manner. Additionally, if it was necessary, the left femoral vein was cannulated with a polyethylene tube (0.28 mm I.D. -0.61 mm O.D., PE10, Japan Becton Dickinson, Tokyo, Japan) and it was connected to an infusion pump (CMA102, CMA Microdialysis AB, Solna, Sweden) to perform the ruthenium red infusion, as described later. During the experiment, the rat was kept warm with heating apparatuses.

Evaluation of Intestinal Cephalexin Absorption Following the preparation, the jejunum and ileum segments started to be perfused independently at a flow rate of 0.2 $\mathrm{ml} / \mathrm{min}$. The perfusate was prepared with Ringer's solution containing cephalexin at a concentration of $500 \mu \mathrm{M}$, and also containing FITC-dextran at the concentration of $1 \mu \mathrm{m}$ as a marker of water flux. The $\mathrm{pH}$ value of the perfusate was adjusted to 6.5 by sodium dihydrogen phosphate solution. ${ }^{27}$ Capsaicin was dissolved in the perfusate at a concentration of 10 or $400 \mu \mathrm{M}$ using diethyl sulfoxide (DMSO) as a solubilizing agent at a final concentration of $1 \%$. DMSO was solely mixed in the perfusate at the same concentration when the control experiments were performed. In several experiments, we used glycylsarcosine as a typical substrate of PEPT1 ${ }^{28,29)}$ It was dissolved in the perfusate at a concentration of $20 \mathrm{~mm}$. This concentration of glycylsarcosine is 50 -fold higher than its Ki value for PEPT1, being sufficient to suppress the transport activity of the transporter. ${ }^{30-32)}$ In addition, ruthenium red was utilized as a non-selective inhibitor of TRP cation channels in this study. ${ }^{33)}$ Ruthenium red was dissolved in saline at a concentration of $1.5 \mathrm{mg} / \mathrm{ml}$, and it was infused into the femoral vein of the rat at a rate of $1 \mathrm{mg} / \mathrm{kg} / \mathrm{h}$ during the equilibrium and experimental periods, if necessary. ${ }^{33)}$

After a 30-min equilibrium period, the fraction collectors were started. The effluents from the jejunum and ileum segments were both collected every $15 \mathrm{~min}$ for $90 \mathrm{~min}$, and the amounts of cephalexin and FITC-dextran in the collected samples were determined as described later. Intestinal cephalexin absorption clearance $\left(C L_{\mathrm{ab}}\right)$ was evaluated as follows ${ }^{27)}$.

$$
\begin{aligned}
& C L_{\text {ab }}=Q \cdot\left(1-\frac{C_{\text {out }}}{C_{\text {in }}}\right) \\
& \frac{C_{\text {out }}}{C_{\text {in }}}=\left(\frac{C_{\text {out , CEX }}}{C_{\text {in, CEX }}}\right) \times\left(\frac{C_{\text {in, FITC }}}{C_{\text {out }, \text { FITC }}}\right)
\end{aligned}
$$

where $C_{\text {out,CEX }}$ and $C_{\text {out,FITC }}$ are the cephalexin and FITC-dextran concentrations in the sample, respectively. $C_{\text {in,CEX }}$ and $C_{\text {in,FITC }}$ are the cephalexin and FITC-dextran concentrations in the perfusate, and $Q$ denotes the perfusion flow rate $(0.2 \mathrm{ml} / \mathrm{min})$.

Assay Methods The cephalexin concentration was determined with an HPLC equipped with an octadodecyl silica column $\left(5 \mu \mathrm{m}, 4.6 \times 150 \mathrm{~mm}\right.$, Superiore ${ }^{\circledR}$ ODS, Shiseido, Tokyo). The samples were filtered with a $0.45-\mu \mathrm{m}$ syringe filter (DISMIC ${ }^{\circledR}-13 \mathrm{HP}$, Advantec, Tokyo, Japan), and 20- $\mu$ 1 of the filtrate was then applied to HPLC. As a mobile phase, $35 \%$ methanol- $65 \%$ ammonium acetate $(10 \mathrm{~mm}, \mathrm{pH} 6.0)$ was used. The flow rate was set at $1.0 \mathrm{ml} / \mathrm{min}$. Cephalexin was determined spectrometrically at a wave length of $270 \mathrm{~nm}$. The detection limit of cephalexin in the sample was $1 \mu \mathrm{m}$. The FITC-dextran concentration was determined with a fluorophotometer (F-2000, Hitachi, Tokyo, Japan) at wave lengths of $485 \mathrm{~nm}$ for excitation and $515 \mathrm{~nm}$ for emission.

Statistics Data are shown as the mean \pm S.E. for $4-5$ experiments. Significant differences between two groups were evaluated by Student's $t$-test. Multiple comparisons were performed by Tukey's $t$-test, if necessary. $p<0.05$ was considered to indicate significance.

\section{RESULTS}

Effects of Capsaicin on Intestinal Cephalexin Absorption in Rats The effects of capsaicin on intestinal cephalexin absorption were examined. In our in situ perfusion experiment, the cephalexin clearance slightly varied through the experimental period regardless of the presence of capsaicin (Fig. 1). Under the control condition, the average $C L_{\mathrm{ab}}$ values were $23.1 \pm 1.6$ and $18.1 \pm 1.1 \mu \mathrm{l} / \mathrm{min}$ in the jejunum and ileum, respectively, indicating that more cephalexin is absorbed in the jejunum than in the ileum in rats (Figs. 1A, B). As shown in Fig. 1A, the average $C L_{\mathrm{ab}}$ value in the jejunum was significantly decreased to $10.8 \pm 1.1 \mu \mathrm{l} / \mathrm{min}$ in the presence of $400 \mu \mathrm{M}$ capsaicin $(p<0.05)$. The average $C L_{\mathrm{ab}}$ value was $20.8 \pm 3.6 \mu \mathrm{l} / \mathrm{min}$ in the presence of $10 \mu \mathrm{M}$ capsaicin, which was not different from the control value (Fig. 1A). The effects of capsaicin on intestinal cephalexin absorption were also observed in the ileum, in which the average $C L_{\mathrm{ab}}$ value was significantly decreased to $9.2 \pm 1.9 \mu \mathrm{l} / \mathrm{min}$ in the presence of $400 \mu \mathrm{M}$ cap- 

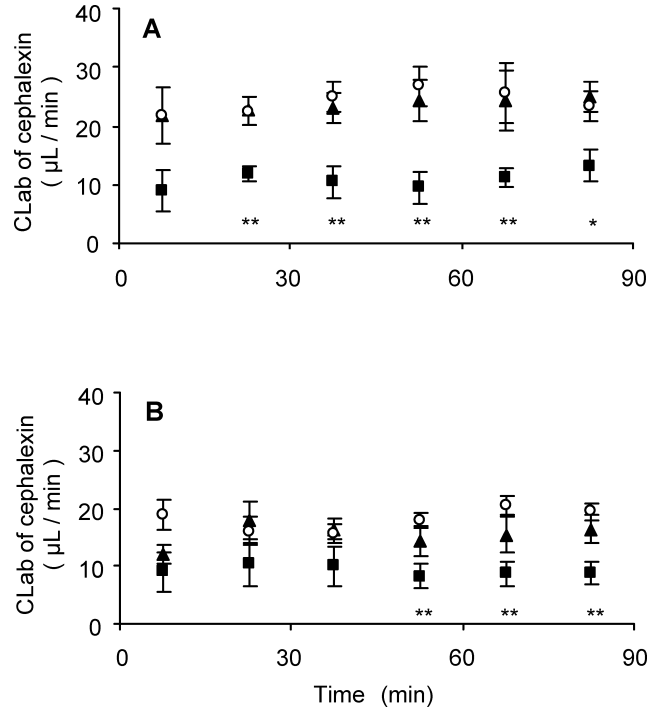

Fig. 1. Intestinal Cephalexin Absorption Clearance $\left(C L_{\mathrm{ab}}\right)$ in the Jejunum (Panel A) and Ileum (Panel B) Measured in the Presence of 0 (Control), 10, and $400 \mu_{\mathrm{M}}$ Capsaicin

Cephalexin was dissolved in the perfusate at a concentration of $500 \mu \mathrm{M}$. The $C L_{\mathrm{ab}}$ values measured under the control condition are indicated with open circles and those values measured in the presence of 10 and $400 \mu \mathrm{M}$ capsaicin are indicated with closed triangles and squares, respectively. Data are shown as the means \pm S.E. of 5 experiments. $* p<0.05, * * p<0.01$; significantly different from the corresponding value in the control group.

saicin $(p<0.05)$ (Fig. 1B). Cephalexin absorption in the ileum was not altered in the presence of $10 \mu \mathrm{M}$ capsaicin (Fig. 1B).

Effects of Ruthenium Red on the Decreased Cephalexin Absorption Caused by Capsaicin

To examine whether the inhibitive effect of capsaicin on intestinal cephalexin absorption was exerted by capsaicin's association with TRPV1, rats were treated with ruthenium red at first, and then intestinal cephalexin absorption was examined in the presence and absence of capsaicin. Ruthenium red itself was shown not to affect intestinal cephalexin $a b-$ sorption under the control condition (Figs. 2A, B). As shown in Fig. 2A, cephalexin absorption in the jejunum was decreased in normal rats in the presence of $400 \mu \mathrm{M}$ capsaicin. However, this inhibitive effect of capsaicin was not observed in rats treated with ruthenium red, where the cephalexin absorption rate was restored from $0.54 \pm 0.07$ to $1.02 \pm 0.05$ $\mathrm{nmol} / \mathrm{min} / \mathrm{cm}$, being comparable to the value under the control condition (Fig. 2A). As shown in Fig. 2B, ruthenium red also seemed to restore the decreased cephalexin absorption caused by $400 \mu \mathrm{M}$ capsaicin in the rat ileum.

Effects of Capsaicin on Glycylsarcosine-Insensitive Intestinal Cephalexin Absorption To clarify to what extent the paracellular permeability of cephalexin is changed when its intestinal absorption process is affected by capsaicin, we examined the effect of capsaicin on glycylsarcosine-insensitive intestinal cephalexin absorption (Fig. 3). It was indicated that glycylsarcosine-insensitive cephalexin absorption contributes to $7.8 \pm 1.4 \%$ of the total cephalexin absorption in the rat jejunum (Fig. 3A). As shown in Fig. 3A, when glycylsarcosine-insensitive cephalexin absorption was evaluated in the presence of capsaicin, the insensitive absorption was increased 6.2 and 4.5 times by 10 and $400 \mu \mathrm{m}$ capsaicin, respectively. The capsaicin-induced increase of the glycylsar-
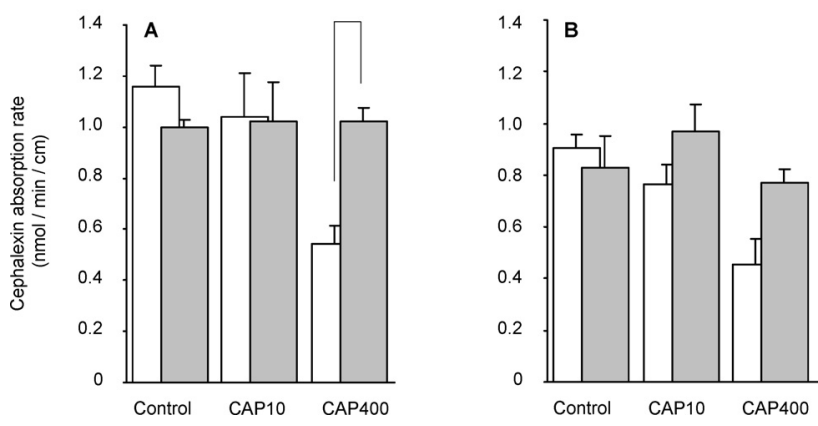

Fig. 2. Effect of Ruthenium Red on Cephalexin Absorption in the Jejunum (Panel A) and Ileum (Panel B) of Rats in the Presence and Absence of Capsaicin

Ruthenium red was intravenously infused into rats at a rate of $1 \mathrm{mg} / \mathrm{kg} / \mathrm{h}$. The cephalexin absorption rates in rats with or without ruthenium red treatment are indicated with closed and open columns, respectively. The experiments performed in the presence of 0 (control), 10, and $400 \mu \mathrm{M}$ capsaicin are denoted as 'Control', 'CAP10', and 'CAP400', respectively. Data are shown as the mean \pm S.E. of $4-5$ experiments. $* p<0.05$; significantly different between the values.
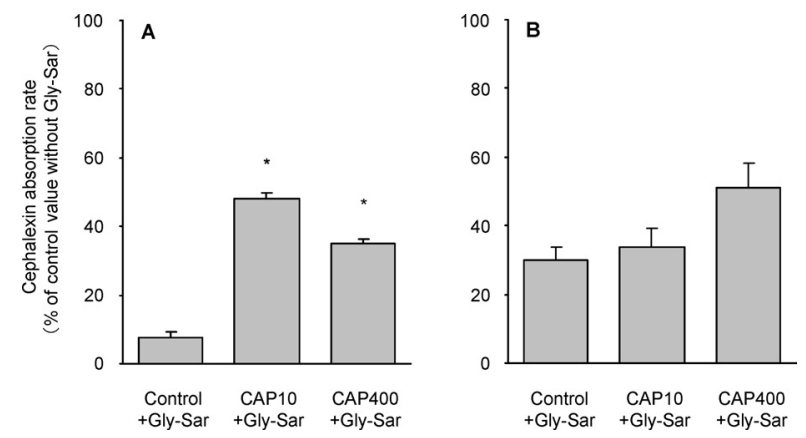

Fig. 3. Effect of Capsaicin on Glycylsarcosine-Insensitive Cephalexin Absorption in the Jejunum (Panel A) and Ileum (Panel B) of Rats

The insensitive cephalexin absorption was evaluated in the presence of $20 \mathrm{~mm}$ glycylsarcosine (Gly-Sar). The experiments performed in the presence of 0 (control), 10, and $400 \mu \mathrm{M}$ capsaicin are denoted as 'Control', 'CAP10', 'CAP400', respectively. Data are shown as the means \pm S.E. of 4 experiments. $* p<0.05$; significantly different from the corresponding control value.

cosine-insensitive cephalexin absorption was also observed in the rat ileum, though the differences did not reach significance (Fig. 3B).

\section{DISCUSSION}

Capsaicin has been used as a pungent food ingredient since ancient times, but it has only recently been revealed that capsaicin associates with TRPV1, and thereby stimulates afferent neurons innervating the gastrointestinal tract.

In this study, we examined whether capsaicin changes intestinal cephalexin absorption in rats, and demonstrated that the intestinal absorption of cephalexin on average was reduced from $23.1 \pm 1.6$ to $10.8 \pm 1.1 \mu \mathrm{l} / \mathrm{min}$ in the presence of $400 \mu \mathrm{M}$ capsaicin in the rat jejunum (Fig. 1A). It was thus obvious that capsaicin affects intestinal cephalexin absorption. In addition, the reduced cephalexin absorption caused by capsaicin was restored when rats were treated with ruthenium red, a non-selective inhibitor of TRP cation channels (Fig. 2). ${ }^{33)}$ Since intestinal cephalexin absorption is mainly mediated by PEPT1, ${ }^{25,26)}$ these findings suggest that PEPT1 activity is altered by capsaicin associating with TRPV1 of afferent neurons innervating the gastrointestinal tract. 
It has been reported that PEPT1 activity is regulated by several intracellular factors, such as the activity of protein kinaseC ${ }^{34)}$ cAMP level, ${ }^{35)}$ or $\mathrm{Ca}^{2+}$ concentration. ${ }^{24)}$ In addition to these factors, an $\mathrm{Na}^{+} / \mathrm{H}^{+}$exchanger is also shown to be a factor determining PEPT1 activity. ${ }^{36}$ That is, the exchanger is demonstrated to regulate the proton gradient across the cellular membrane, which PEPT1 requires as a driving force for its $\mathrm{H}^{+}$/peptide co-transporting function. ${ }^{36)}$ Moreover, the activity of the $\mathrm{Na}^{+} / \mathrm{H}^{+}$exchanger in intestinal epithelia is suggested to be controlled by the afferent neurons innervating the gastrointestinal tract, because it was demonstrated that the $\mathrm{Na}^{+} / \mathrm{H}^{+}$exchanger-mediating recovery of intracellular $\mathrm{pH}$ after the experimental acidification was retarded by VIP, a polypeptide hormone released from the nerve endings of afferent neurons. ${ }^{37)}$ It was also reported that cellular uptake of the PEPT1 substrate glycylsarcosine was decreased in the presence of VIP. ${ }^{37)}$ With these findings, we speculated about mechanisms responsible for the altered cephalexin absorption clearance as follows: Capsaicin stimulates the afferent neurons by associating with TRPV1 and the neurons release hormones affecting the gastrointestinal tract such as VIP. VIP modulates the $\mathrm{Na}^{+} / \mathrm{H}^{+}$exchanger on the epithelial cells and the proton gradient across the cellular membrane is altered. Since the $\mathrm{H}^{+}$/peptide co-transporting activity of PEPT1 is thereby affected, intestinal cephalexin absorption is decreased.

As for intestinal cephalexin absorption, it has been considered that the paracellular pathway also contributes to the absorption process in addition to the transcellular pathway mediated by PEPT $1 .{ }^{25,26)}$ Therefore, we examined to what extent the cephalexin absorption process via the paracellular pathway is changed in the presence of capsaicin. As glycylsarcosine is a representative substrate of PEPT1 which competes with cephalexin absorption mediated by PEPT1, the contribution of the paracellular pathway can be evaluated by measuring cephalexin absorption in the presence of an excess amount of glycylsarcosine. ${ }^{28,29,32)}$ As shown in Fig. 3A, since the cephalexin absorption rate was $7.8 \pm 1.4 \%$ of the control value in the presence of $20 \mathrm{~mm}$ glycylsarcosine, the contribution of the paracellular pathway was considered to be less than $10 \%$ of the total cephalexin absorption in the rat jejunum. When we evaluated glycylsarcosine-insensitive cephalexin absorption in the rat jejunum, it was increased 6.2 and 4.5 times in the presence of 10 and $400 \mu \mathrm{m}$ capsaicin, respectively (Fig. 3A). It was thus revealed that the paracellular permeability of cephalexin was increased by capsaicin. The precise mechanism underlying the increased paracellular permeability of cephalexin is currently unclear. However, since the paracellular permeability is altered according to the intracellular $\mathrm{Ca}^{2+}$ concentration, ${ }^{23)}$ it is probable that capsaicin has some effects to change the intracellular $\mathrm{Ca}^{2+}$ concentration. The change in the $\mathrm{Ca}^{2+}$ concentration may be caused by VIP released from afferent neurons by capsaicin's association with TRPV1. In addition, TRPV1 is known to regulate extracellular $\mathrm{Ca}^{2+}$ influx, and it has been suggested to be expressed in gastrointestinal tissues. ${ }^{6,19,20,22)}$ Therefore, the change in the $\mathrm{Ca}^{2+}$ concentration may be also brought about by capsaicin directly affecting intestinal epithelial cells. Further investigations are necessary to clarify the mechanisms responsible for the increased paracellular permeability of cephalexin caused by capsaicin.
In conclusion, we indicated that capsaicin alters both transcellular and paracellular pathways of intestinal cephalexin absorption. We demonstrated that capsaicin primarily decreases cephalexin absorption mediated via PEPT1 by associating with TRPV1, and that capsaicin also increases the paracellular permeability of cephalexin in the rat jejunum. These findings provide helpful information to avoid undesirable drug/food interactions.

Acknowledgment This work was supported by a Grantin-Aid for Scientific Research from the Japan Society for the Promotion of Sciences.

\section{REFERENCES}

1) Limlomwongse L., Chaitauchawong C., Tongyai S., J. Nutr., 109, $773-777$ (1979)

2) Abdel Salam O. M., Szolcsanyi J., Porszasz R., Mozsik G., Eur. J. Pharmacol., 305, 127-136 (1996).

3) Caterina M. J., Schumacher M. A., Tominaga M., Rosen T. A., Levine J. D., Julius D., Nature (London), 389, 816-824 (1997).

4) Szallasi A., Blumberg P. M., Pharmacol. Rev., 51, 159-212 (1999).

5) Tominaga M., Caterina M. J., Malmberg A. B., Rosen T. A., Gilbert H., Skinner K., Raumann B. E., Basbaum A. I., Julius D., Neuron, 21 $531-543$ (1998)

6) Clapham D. E., Montell C., Schultz G., Julius D., Pharmacol. Rev., 55, 591-596 (2003).

7) Holzer P., Pharmacol. Rev., 43, 143-201 (1991).

8) Cooke H. J., Ann. N.Y. Acad. Sci., 657, 313-318 (1992).

9) Nuki C., Kawasaki H., Kitamura K., Takenaga M., Kangawa K., Eto T., Wada A., Biochem. Biophys. Res. Commun., 196, 245-251 (1993).

10) Odes H. S., Muallem R., Reimer R., Beil W., Schwenk M., Sewing K. F., Am. J. Physiol., 265, G270-G276 (1993).

11) Reddix R., Kuhawara A., Wallace L., Cooke H. J., J. Pharmacol. Exp. Ther, 269, 1124-1129 (1994).

12) Lambrecht N., Burchert M., Respondek M., Muller K. M., Peskar B. M., Gastroenterology, 104, 1371-1380 (1993).

13) Holzer P., Gastroenterology, 114, 823-839 (1998).

14) Bartho L., Holzer P., Naunyn Schmiedebergs Arch. Pharmacol., 353, 102-109 (1995).

15) Buck S. H., Burks T. F., Pharmacol. Rev., 38, 179-226 (1986).

16) Nassar C. F., Barada K. A., Abdallah L. E., Hamdan W. S., Taha A. M., Atweh S. F., Saade N. E., Am. J. Physiol., 268, G695-699 (1995).

17) Barada K. A., Saade N. E., Atweh S. F., Nassar C. F., Am. J. Physiol., 275, G822-G888 (1998).

18) Barada K. A., Saade N. E., Atweh S. F., Khoury C. I., Nassar C. F., Regul. Pept., 90, 39-45 (2000).

19) Nozawa Y., Nishihara K., Yamamoto A., Nakano M., Ajioka H., Matsuura N., Neurosci. Lett., 309, 33-36 (2001).

20) Kato S., Aihara E., Nakamura A., Xin H., Matsui H., Kohama K., Takeuchi K., Biochem. Pharmacol., 66, 1115-1121 (2003).

21) Ward S. M., Bayguinov J., Won K. J., Grundy D., Berthoud H. R., J. Comp. Neurol., 465, 121-135 (2003).

22) Kechagias S., Botella S., Petersson F., Borch K., Ericson A. C., Scand. J. Gastroenterol., 40, 775-782 (2005).

23) Jovov B., Lewis S. A., Crowe W. E., Berg J. R., Wills N. K., Am. J. Physiol., 266, F775-F784 (1994).

24) Wenzel U., Kuntz S., Diestel S., Daniel H., Antimicrob. Agents Chemother, 46 1375-1380 (2002).

25) Yamashita S., Yamazaki Y., Mizuno M., Masada M., Nadai T., Kimura T., Sezaki H., J. Pharmacobio-Dyn., 4, 227-233 (1984).

26) Terada T., Saito H., Mukai M., Inui K., Am. J. Physiol., 273, F706F711 (1997).

27) Chu X. Y., Sanchez-Castano G. P., Higaki K., Oh D. M., Hsu C. P., Amidon G. L., J. Pharmacol. Exp. Ther., 99, 575-582 (2001).

28) Ganapathy M. E., Huang W., Wang H., Ganapathy V., Leibach F. H., Biochem. Biophys. Res. Commun., 246, 470-475 (1998).

29) Irie M., Terada T., Okuda M., Inui K., Pflügers Arch., 449, 186-194 (2004).

30) Moore V. A., Irwin W. J., Timmins P., Chong S., Dando S. A., Morrison R. A., Int. J. Pharm., 210, 15-27 (2000) 
31) Buyse M., Berlioz F., Guilmeau S., Tsocas A., Voisin T., Peranzi G., Merlin D., Laburthe M., Lewin M. J., Roze C., Bado A., J. Clin. Invest., 108, 1483-1494 (2001).

32) Putnam W. S., Pan L., Tsutsui K., Takahashi L., Benet L. Z., Pharm. Res., 19, 27-33 (2002).

33) Tashima K., Nakashima M., Kagawa S., Kato S., Takeuchi K., Med. Sci. Monit., 8, BR157-BR163 (2002).

34) Brandsch M., Miyamoto Y., Ganapathy V., Leibach F. H., Biochem. J.,
299, 253-260 (1994).

35) Muller U., Brandsch M., Prasad P. D., Fei Y. J., Ganapathy V., Leibach F. H., Biochem. Biophys. Res. Commun., 218, 461-465 (1996).

36) Ganapathy V., Leibach F. H., Biochim. Biophys. Acta, 816, 234-240 (1985).

37) Anderson C. M. H., Mendoza M. E., Kennedy D. J., Raldua D., Thwaites D. T., Br. J. Pharmacol., 138, 564-573 (2003). 